\title{
Article \\ Measurement of Thinned Water-Cooled Wall in a Circulating Fluidized Bed Boiler Using Ultrasonic and Magnetic Methods
}

\author{
Jinyi Lee ${ }^{1,2, *(\mathbb{D}}$, Eunho Choe ${ }^{3}$, Cong-Thuong Pham ${ }^{4}\left(\mathbb{D}\right.$ and Minhhuy Le ${ }^{4,5, * \mathbb{C}}$ \\ 1 Department of Electronic Engineering, Chosun University, Gwangju 61452, Korea \\ 2 Interdisciplinary Program in IT-Bio Convergence System, Chosun University, Gwangju 61452, Korea \\ 3 Department of Control and Instrumentation, Graduate School of Chosun University, Gwangju 61452, Korea; \\ fordo2@nate.com \\ 4 Faculty of Electrical and Electronic Engineering, Phenikaa University, Hanoi 12116, Vietnam; \\ thuong.phamcong@phenikaa-uni.edu.vn \\ 5 A\&A Green Phoenix Group JSC, Phenikaa Research and Technology Institute (PRATI), No.167 Hoang Ngan, \\ Trung Hoa, Cau Giay, Hanoi 11313, Vietnam \\ * Correspondence: jinyilee@chosun.ac.kr (J.L.); huy.leminh@phenikaa-uni.edu.vn (M.L.)
}

Citation: Lee, J.; Choe, E.; Pham, C.-T.; Le, M. Measurement of Thinned Water-Cooled Wall in a Circulating Fluidized Bed Boiler Using Ultrasonic and Magnetic Methods. Appl. Sci. 2021, 11, 2498. https://doi.org/ 10.3390/app11062498

Academic Editor:

Giuseppe Lacidogna

Received: 26 January 2021

Accepted: 8 March 2021

Published: 11 March 2021

Publisher's Note: MDPI stays neutral with regard to jurisdictional claims in published maps and institutional affiliations.

Copyright: () 2021 by the authors. Licensee MDPI, Basel, Switzerland. This article is an open access article distributed under the terms and conditions of the Creative Commons Attribution (CC BY) license (https:// creativecommons.org/licenses/by/ $4.0 /)$.

\begin{abstract}
In this paper, a nondestructive inspection system is proposed to detect and quantitatively evaluate the size of the near- and far-side damages on the tube, membrane, and weld of the watercooled wall in the fluidized bed boiler. The shape and size of the surface damages can be evaluated from the magnetic flux density distribution measured by the magnetic sensor array on one side from the center of the magnetizer. The magnetic sensors were arrayed on a curved shape probe according to the tube's cross-sectional shape, membrane, and weld. On the other hand, the couplant was doped to the water-cooled wall, and a thin film was formed thereon by polyethylene terephthalate. Then, the measured signal of the flexible ultrasonic probe was used to detect and evaluate the depth of the damages. The combination of the magnetic and ultrasonic methods helps to detect and evaluate both near and far-side damages. Near-side damages with a minimum depth of $0.3 \mathrm{~mm}$ were detected, and the depth from the surface of the far-side damage was evaluated with a standard deviation of $0.089 \mathrm{~mm}$.
\end{abstract}

Keywords: circulating fluidized bed combustion boiler; water-cooled wall tube; magnetic sensor array; magnetic flux density; flexible ultrasonic probe

\section{Introduction}

Circulating fluidized bed combustion boilers burn various fuels such as wood, coal, and combustible waste together with solid fluidized media such as sand and ash [1]. In addition, combustion air is injected at high speed through a distribution plate at the bottom of the furnace to burn coal in a gas-solid flow condition inside the furnace. The high temperature of the heated fluid medium particles scatter and circulate in a suspended state to transfer heat to the heat transfer tube. Since heat is transferred through collisional contact with the fluid particles, the heat transfer coefficient is very superior compared to the convection heat exchange method of the existing boiler. However, due to the collision contact between the surface of the water-cooled wall and the fluid particles, which is repeated as the operation time elapses, erosion due to direct exposure to combustion flames, corrosion due to high-temperature combustion and formation of potassium chloride, and acceleration of corrosion due to adhesion could appear. Thus, the life cycle of the watercooled wall is shorter than that of the existing boiler systems. In addition, the lower part where the concentration of the fluid medium is high is a splash area where the fluid medium violently behaves, and the water-cooled wall is severely damaged. These damages intensify in the kick-out area located at the boundary between the lower fireproof part and the water-cooled wall [2]. On the other hand, when abrasion and corrosion occur on the 
water-cooled wall tube, leakage and secondary damage due to the leakage may occur. It is also very important to periodically monitor and maintain the thickness of the water-cooled wall since damage to the tube, membrane, and welding portion of the water-cooled wall can cause a decrease in power generation efficiency.

Recently, numerous NDT methods have been developed for the inspection of damage on the water-cooled wall. Ultrasonic testing (UT) applies an acoustic medium to the inspection area of the water-cooled wall and measures the change in the reflection time of the ultrasonic wave according to the thickness change when the ultrasonic wave is incident [3]. Although it is possible to perform a precise inspection of the inner surface of the tube, it is difficult to measure the surface corrosion, and the incident angle of ultrasonic waves may vary according to the manual inspection of the operator, resulting in an error in thickness measurement. Phased array ultrasonic testing (PAUT) has been developed to reduce mechanical errors while scanning the probe on the specimens and signal enhancement. It provides excellent results of damage detection and quantitative evaluation of the damage size, such as depth and length $[4,5]$. However, the UT and PAUT methods require continuous supplement of the coupling materials such as water for the propagation of ultrasonic wave between the probes and the tube, and the surface of the tube should be cleaned before the inspection. Therefore, it is difficult to operate in the inspection of the water-cooled wall tubes in the power generation, and it also requires high technical trained operators to use the UT and PAUT systems. Electromagnetic testing methods, including eddy current testing (ECT), remote field eddy current testing (RFECT), and magnetic flux leakage testing (MFLT), are the fast, reliable, and easy operation methods for the inspection of damages in the tubes. These are non-contact inspection methods that do not require the coupling material during the inspection. ECT is a widely used method for the inspection of heat exchanger tubes and boilers of nuclear power plants [6-11]. This method is highly sensitive to the surface cracks, but it is limited to detecting deep defects due to the high concentration of eddy current on the specimen surface in the skin depth effect. Especially, the eddy current has more difficulty penetrating the wall thickness of the water-cooled tube because it has high magnetic permeability. RFECT [12-16] uses a probe consisting of an excitation coil and a measuring coil that can be inserted into a ferromagnetic heat pipe tube such as the water-cooled tubes. The magnetic energy generated by the interpolation type excitation coil goes from the excitation coil to the outside of the tube and flows in the axial direction, and then back to the inside from the remote field area at a certain distance. The measuring coil can sense the energy delivered without receiving it from the excitation coil. In order to increase the ratio of the signal to noise, it is necessary to increase the cross-sectional area and the number of turns of the excitation coil and the measurement coil so that the spatial resolution of the probe is low. Therefore, there is a limitation in quantitatively evaluating where the damage is occurring on the water-cooled wall tube, the weld, and the membrane. For further improvement of the sensitivity, a giant magnetoresistance (GMR) and Hall sensors were used to measure the low magnetic leakage signal in the MFLT systems $[17,18]$. This method makes it possible to detect a defect on the surface and near the surface of the water-cooled wall tubes. However, it is still difficult to measure the thickness changes of the tube due to the damages. The combination of the ultrasonic and electromagnetic field has been developed in the electromagnetic acoustic transducer (EMAT) system for the inspection of the water-cooled tubes $[19,20]$. The magneto-elastic phenomenon and Lorentz force help the EMAT inspect a deeper damage without the need for coupling material. However, the signal is weak and requires advanced signal processing circuits and algorithms. In addition, the EMAT probe has a big size, and thus, it is not efficient to build an array EMAT probe with a high spatial resolution for quantitative evaluation of damage sizes.

This study proposed a combination of the magnetic flux leakage testing and ultrasonic testing methods for the efficient detection and quantitative evaluation of the depth and residual thickness distribution of the near-side and far-side corrosion of the water-cooled wall. A Hall sensor array probe with 48 elements arrayed in an interval of $2.5 \mathrm{~mm}$ was 
developed to detect the near-side damages and thus make it possible to evaluate the damage size. A flexible ultrasonic probe (FUP) was developed to detect the far-side damages on the tube, membrane, and welding lines of the water-cooled wall. The FUP was incorporated with a flexible membrane that allows the transmission of the ultrasonic wave from the probe to the water-cool tube surface efficiently. Thus, it is not required to largely supply coupling material during the inspection. In addition, the FUPs could be arrayed according to the water-cool plates for fast inspection. For the verification of the proposed method, artificial tapper-type wears and slit-type damages with different sizes were produced on the tube, membrane, and welding lines of the water-cooled wall. Both the detection and size/depth evaluation of the damages will be discussed.

\section{Materials and Methods}

\subsection{Measurement of Magnetic Flux Density}

Figure 1 shows the simplified 2D dipole model of the thickness changing on the boiler water-cooled wall tube due to corrosion [21,22]. A U-type magnetizer is placed on the surface of the membrane. The width, distance between poles, and height of the magnetizer are expressed as $W, D$, and $H$, respectively. The corrosion depth and length are $d$ and $D / 2+W$, respectively. The distance between the magnetizer and the specimen, i.e., lift-off, is expressed as $h$. Then, the lift-off at the corrosion is $h+d$. In the dipole model, magnetic charges $\pm m$ per unit area are assumed to be distributed along the length of the magnetizer poles, membrane length, and corrosion length according to the assumption in the dipole model [21,22]. The magnetic flux density in the $y$-axis direction at the position of $P\left(x_{p}, y_{p}\right)$ is the summary of the magnetic field produced from the magnetic charges, as expressed in Equation (1). The vertical magnetic field from the left magnetizer pole, right magnetizer pole, no-corrosion specimen length, and corrosion specimen length are expressed as $H_{L U}$, $H_{R U}, H_{L D}, H_{R D}$ in Equations (2)-(5), respectively.

$$
\begin{gathered}
H_{y}=H_{L U}+H_{L D}+H_{R U}+H_{R D} \\
H_{L U}=\frac{+m}{4 \pi \mu} \int_{-\frac{D}{2}-W}^{\frac{D}{2}} \frac{y_{p}}{\left\{\left(x_{p}-u\right)^{2}+\left(y_{p}\right)^{2}\right\}^{\frac{3}{2}}} d u \\
H_{R U}=\frac{-m}{4 \pi \mu} \int_{\frac{D}{2}}^{\frac{D}{2}+W} \frac{y_{p}}{\left\{\left(x_{p}-u\right)^{2}+\left(y_{p}\right)^{2}\right\}^{\frac{3}{2}}} d u \\
H_{L D}=\frac{-m}{4 \pi \mu} \int_{-\frac{D}{2}-W}^{0} \frac{\left(y_{p}+h\right)}{\left\{\left(x_{p}-u\right)^{2}+\left(y_{p}+h\right)^{2}\right\}^{\frac{3}{2}}} d u \\
H_{R D}=\frac{+m}{4 \pi \mu} \int_{0}^{\frac{D}{2}+W} \frac{\left(y_{p}+h+d\right)}{\left\{\left(x_{p}-u\right)^{2}+\left(y_{p}+h+d\right)^{2}\right\}^{\frac{3}{2}}} d u
\end{gathered}
$$




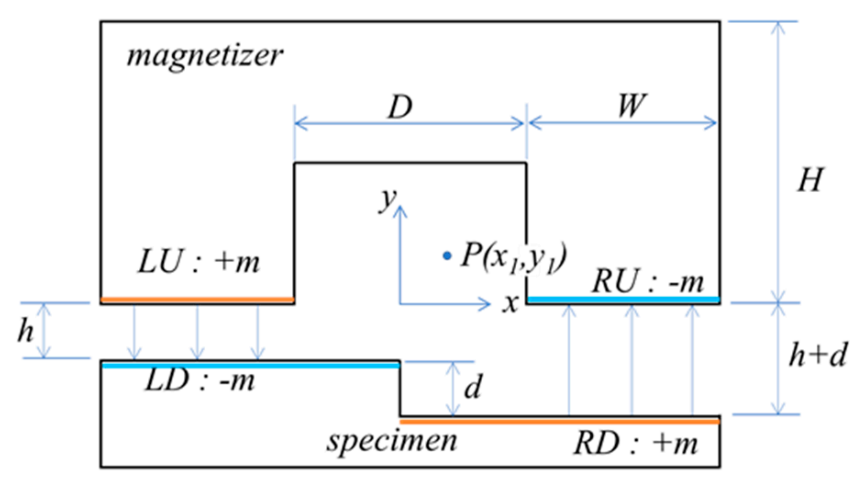

$L U, L D, R U, R D$ express

left-upper, left-down, right-upper, right-down sides.

Figure 1. 2D dipole model of magnetic flux leakage testing system for wall thinning of the boiler water-cooled tube.

Figures 2 and 3 show the result of calculating $H_{y}$ for the depth of the defect $d$ in the range of $0 \sim 3 \mathrm{~mm}$ and position $x_{p}$ in the range of $-15-15 \mathrm{~mm}$ using Equations (1)-(5); where $m$ was assumed as $2 \pi \times 10^{-4}[\mathrm{H} / \mathrm{m}]$, relative magnetic permeability of the ferromagnetic material $\mu=500$, the lift-off $h=1 \mathrm{~mm}$, and the width $(D)$ and width $(W)$ of the magnetic poles were assumed to be $10 \mathrm{~mm}$. Large changes of the magnetic flux intensity on the defect size with different depths are shown in Figure 2. It is noted that the $H_{y}$ has small changes at the center position of the magnetizer and increases as closer to the pole of the magnetizer. Thus, it should not position the magnetic sensor at the center of the magnetizer. Furthermore, Figure 3 shows the relationship between the $H_{y}$ with the defect's depth at a different position on the $x$-axis. It shows a less sensitivity of the $H_{y}$ to the defect's depth when placing the sensor at the center of the magnetizer $(x=0)$, and a similar-high sensitivity when the sensor is at $2-5 \mathrm{~mm}$ from the magnetizer's center. However, the closer to the magnetizer's pole, the higher the intensity of the magnetic flux that could saturate the magnetic sensor. Therefore, the results suggest positioning the sensor at a distance of $2 \mathrm{~mm}$ where the sensitivity to the defect's depth is high, and the magnetic flux density is hard to saturate the magnetic sensor.

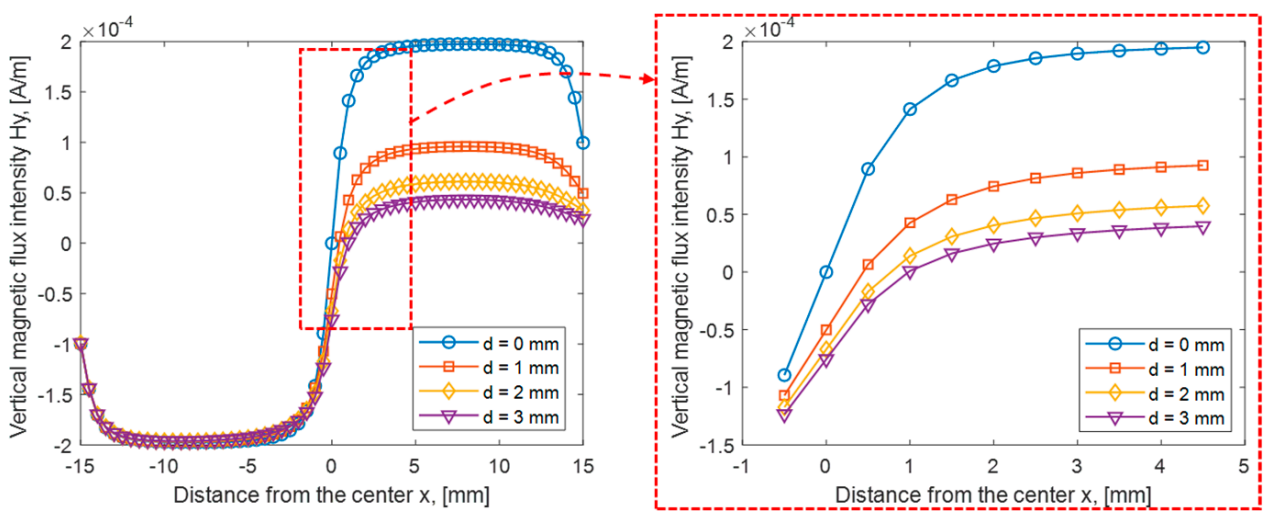

Figure 2. Simulation result with magnetic dipole model for different depth $d$. 

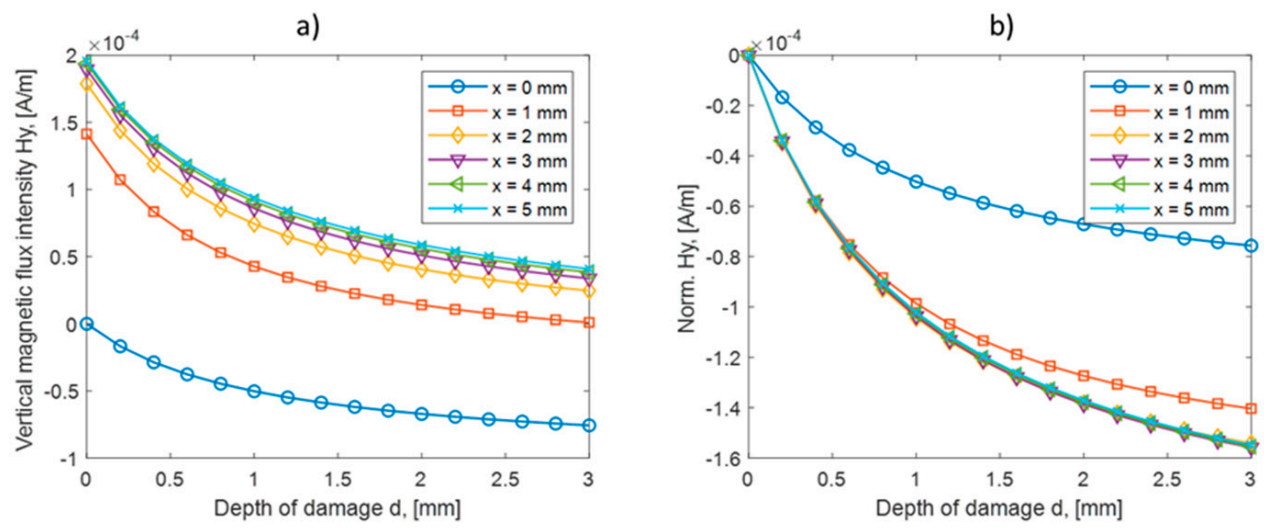

Figure 3. (a) Relationship between the depth of damage $d$ and the vertical component of magnetic flux intensity $H_{y}$ and (b) the normalized $H_{y}$ at different positions from the yoke's center in the $x$-axis.

From the results in Figure 3, it is possible to estimate the relationship between the $H_{y}$ and $d$ by a quadratic equation, as shown in Equation (6). Here, $\alpha_{1}, \alpha_{2}$, and $\alpha_{3}$ are constants. On the other hand, when using the Hall sensor, the magnetic flux density in the vertical direction can be measured by the Hall sensor output voltage $V_{H}$, as expressed in Equation (7) (relative permeability of the air is assumed to 1):

$$
\begin{aligned}
& H_{y}=\alpha_{1}\left(d-\alpha_{2}\right)^{2}+\alpha_{3} \\
& V_{H}=k I B \cos \theta=k I H_{y}
\end{aligned}
$$

where $V_{H}, k, B, I, \theta$ denote the Hall voltage, the Hall constant, the magnetic flux density incident on the Hall sensor, the Hall sensor input current, and the direction angle of the magnetic flux density perpendicularly incident on the Hall sensor. The Hall voltage $V_{H}$ by Equation (7) is linearly proportional to $H y$, the magnetic flux density in the vertical direction. On the other hand, if the Hall constant $(k)$ and the Hall sensor input current $(I)$ are constant, and Equation (6) is substituted into Equation (7), it is expressed as Equations (8) and (9). That is, by measuring the magnetic flux density in the vertical direction, the depth of the defect can be quantitatively evaluated, where $c_{1}$ and $c_{2}$ are constants:

$$
\begin{gathered}
V_{H}=k I\left\{a_{1}\left(d+a_{2}\right)^{2}+a_{3}\right\} \\
d=\sqrt{c_{1} V_{H}+c_{2}}-a_{2}
\end{gathered}
$$

Figure 4 shows the block diagram of the signal processing for a single Hall sensor element. The output voltage of the Hall sensor $V_{H}$ was low-pass filtered (LPF) to remove the high-frequency noise signal. The first stage amplifier was used to gain the signal before transferring to the main signal processing circuits. The LPFs and first stage amplifier were attached near to the Hall sensor in the sensor probe. The second stage amplifier has a controllable gain, which was adjusted by the software in the PC. The signal was then converted to digital via A/D converter and real-time display/process in the PC. The proposed inspection system uses multiple Hall sensors; thus, the number of LPFs, first amplifiers and second amplifiers are the same as the number of Hall sensors for simultaneous signal processing. 


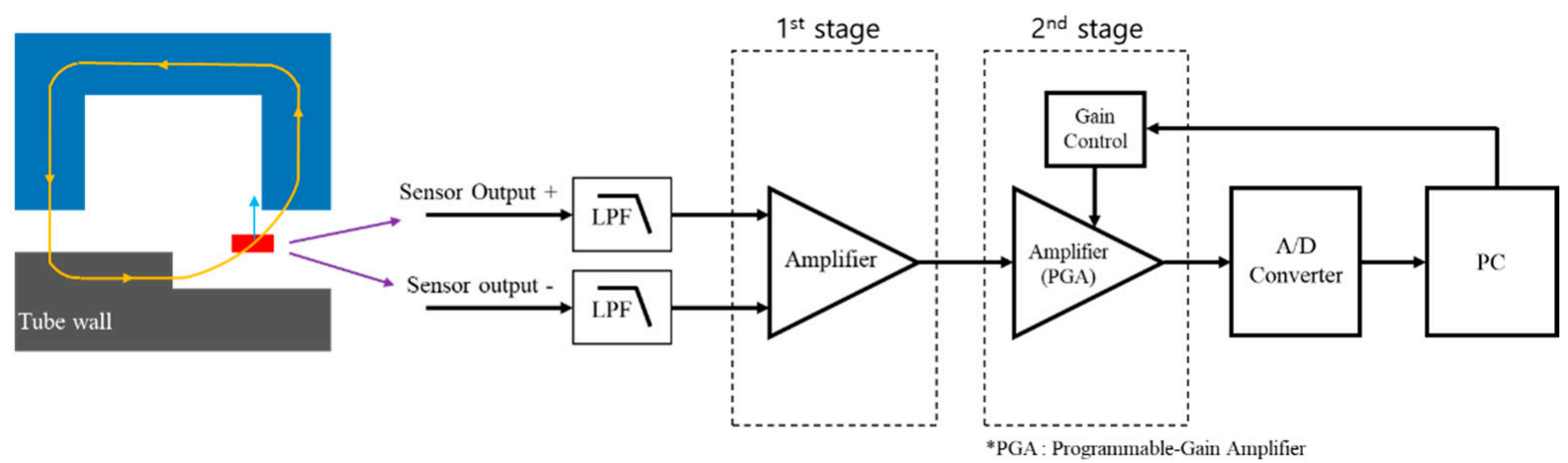

Figure 4. Signal processing block diagram of the magnetic flux leakage testing system. The block diagram is for a single Hall sensor.

Figure 5 shows the proposed magnetic leakage testing system to inspect the corrosion in the water-cooled tube wall. A magnetic sensor array and magnetizer were manufactured to fit with the water-cooled tube's surface, as shown in the left and middle drawing. The magnetic sensor array was placed at the middle of magnetizer poles for measuring the distribution of vertical magnetic flux, as discussed in the previous paragraphs. There were three wheels (a front and two rears wheels) used to maintain the lift-off between the sensor and the tube and help scan the tube easily.
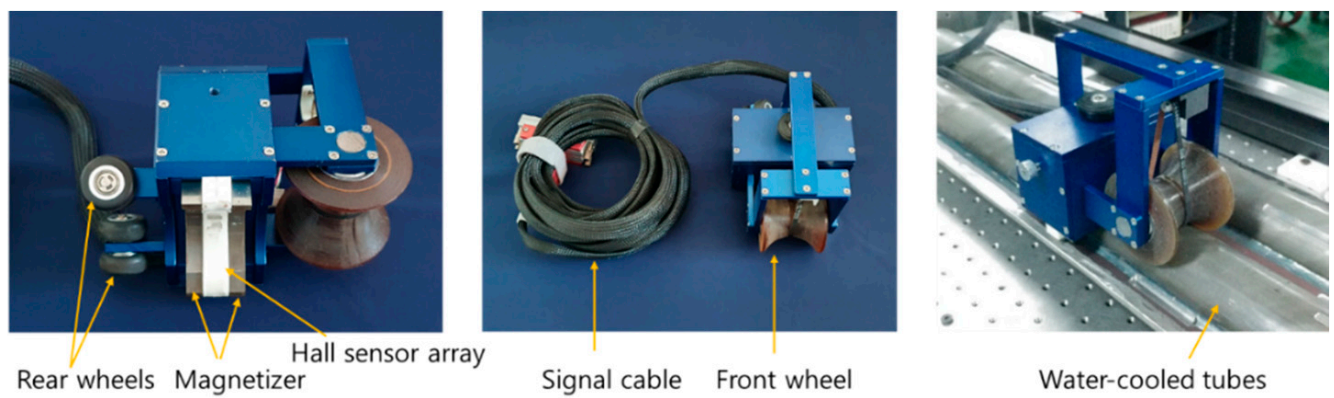

Figure 5. Configurations of the magnetic flux leakage testing system.

\subsection{Flexible Ultrasonic Testing}

Ultrasonic probe usually requires a supplement of a coupling material for transmitting ultrasonic wave from the transducer to the test specimen. It complicates the inspection system and is waste of coupling material. In addition, the test specimen surface should be flat enough to maintain a positive lift-off (non-contact) for protecting the collision of the transducer with the test specimen. It is hard for the inspection of near-surface defects in the water-cooled tube because the changes of the tube wall could make an unpredictable lift-off that could lead to the collision and break the transducer. Therefore, we propose using a flexible transducer that the lift-off could be varied and not require using the coupling material [23]. At the head of a normal transducer, we attached a flexible membrane that was water-filled. The membrane has a sphere shape after filing the water and maintains contact with the tube even though the lift-off can vary. Also, the ultrasound wave can still propagate from the transducer to the water membrane and come to the test specimen.

A sample flexible transducer is shown in Figure 6a. The transducer has a spring that keeps the contact between the membrane with the test specimen during the scan. The received time-domain signal of the transducer, which is A-scan signal $(u(t))$, is shown in Figure $6 \mathrm{~b}$. For a better signal-to-noise ratio, the spectrogram of the A-scan signal was processed $(S(\tau, f))$ and extracted only the signal $\left(S_{A}(\tau)\right)$ at the center frequency of the transducer $\left(f_{c}\right)$, as shown in Figure $6 \mathrm{c}$,d. The spectrogram $(S(\tau, f))$ and extracted signals $\left(S_{A}(\tau)\right)$ are calculated as expressed in Equations (10) and (11), respectively; where, $h$ is a 
sliding Gaussian window. The extracted signal $\left(S_{A}(\tau)\right)$ was then stacked to form the B-scan signal while scanning the transducer along with the test specimen.

$$
\begin{gathered}
S(\tau, f)=\left|\int_{-\infty}^{\infty} u(t) h(t-\tau) e^{-j 2 \pi f t} d t\right|^{2} \\
S_{A}(\tau)=\left.S(\tau, f)\right|_{f=f_{c}}
\end{gathered}
$$

a)

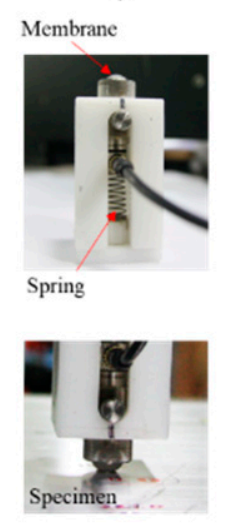

b)

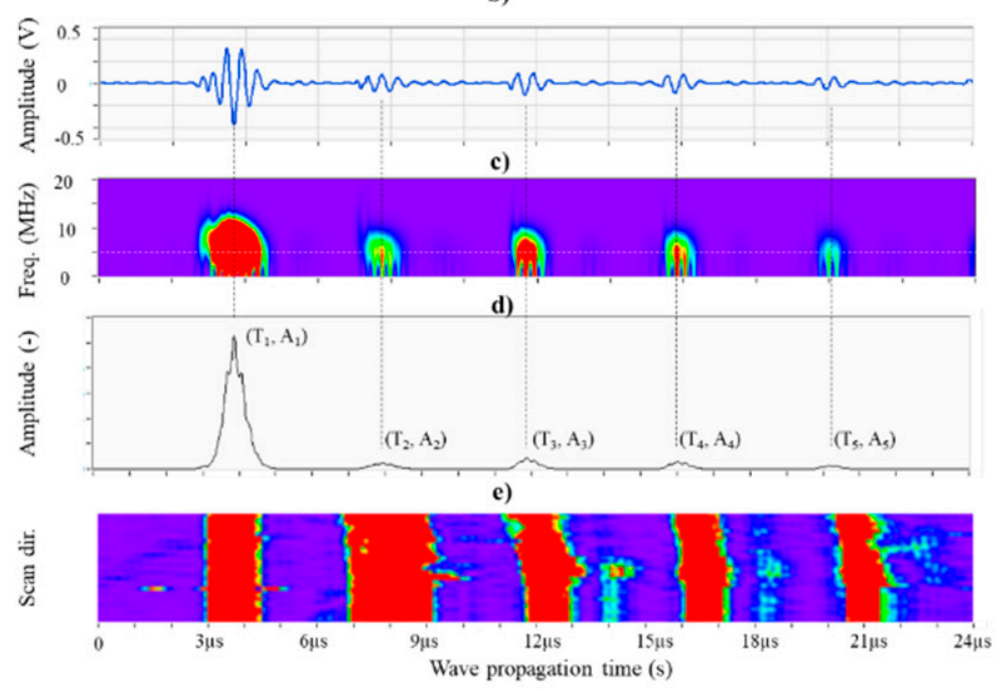

Figure 6. (a) A single flexible ultrasonic transducer measuring a thickness of a specimen, (b) its time-domain signal, (c) spectrogram signal, (d) the cross-section view signal of the spectrogram, and (e) the stacked cross-section view signal (B-scan).

It is observed from the ultrasonic transducer signal that there are multiple peaks. The first peaks are the reflected wave from the specimen surface. It has a delay time of about $4 \mu \mathrm{s}\left(t_{1}, u_{1}\right)$, which is the propagation time from within the probe membrane. This delay time could be varied due to the flexibility of the membrane (lift-off). Thus, it is necessary to eliminate this delay time by shifting the signal with an amount of time $-t_{1}$. In addition, there are four peaks $\left(t_{2}, u_{2}\right),\left(t_{3}, u_{3}\right),\left(t_{4}, u_{4}\right),\left(t_{5}, u_{5}\right)$ next to the specimen surface peak $\left(t_{1}, u_{1}\right)$, which correspond to the repetitions from the bottom surface of the specimen. The time intervals of these four peaks are the same and can be used to calculate the specimen thickness, as shown in Equation (12); where $v$ is the speed of the ultrasound in the specimen.

$$
d=\left(t_{2}-t_{1}\right) \times \frac{v}{2}=\left(t_{3}-t_{2}\right) \times \frac{v}{2}=\cdots=\left(t_{5}-t_{4}\right) \times \frac{v}{2}
$$

Figure 7 is a schematic of the flexible ultrasonic probe (FUP) for quantitatively measuring the specimen thickness. The FUP is an array of multiple transducers (i.e., 6) arranged for covering the tube wall, welding lines, and specimen membrane area. The FUP could be alternated the magnetizer and magnetic sensor array modules in Figure 5. 

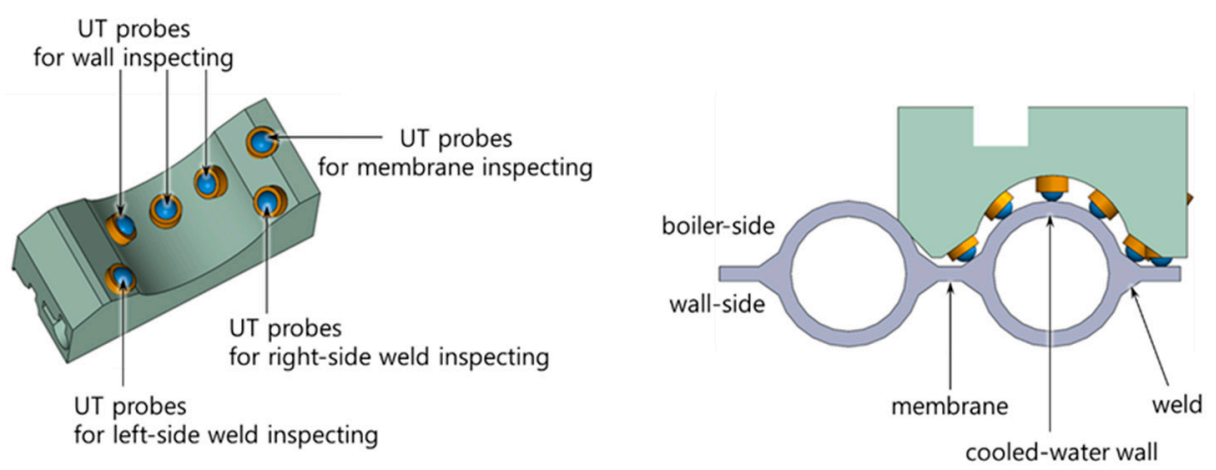

Figure 7. Schematic of the flexible ultrasonic probe (FUP) for measuring the water-cooled wall thicknesses.

\section{Experiment and Results}

\subsection{Specimen}

Figure 8 shows the shape and location of damages on a specimen. A total of four watercooled tubes (SA210C) with inner and outer diameters of 47.3 to $51.3 \mathrm{~mm}$ and $63.5 \mathrm{~mm}$, respectively, one-sided (t11-t14) and double-sided artificial damages (t41-44) simulated for wears were produced on Tube- 1 and Tube-4, respectively. In Tube-2, slit-type artificial damages ( $\mathrm{t} 21-\mathrm{t} 28)$ with the same width of $7.0 \mathrm{~mm}$, depth of $0.9 \mathrm{~mm}$, and lengths from 20 to $100 \mathrm{~mm}$ were produced. In Tube-3, slit-type artificial damages (t31-t38) having the same width of $7 \mathrm{~mm}$ and length of $60 \mathrm{~mm}$ and different depths from 0.3 to $3.1 \mathrm{~mm}$ were produced. The detailed location and size are as shown in Tables 1 and 2.

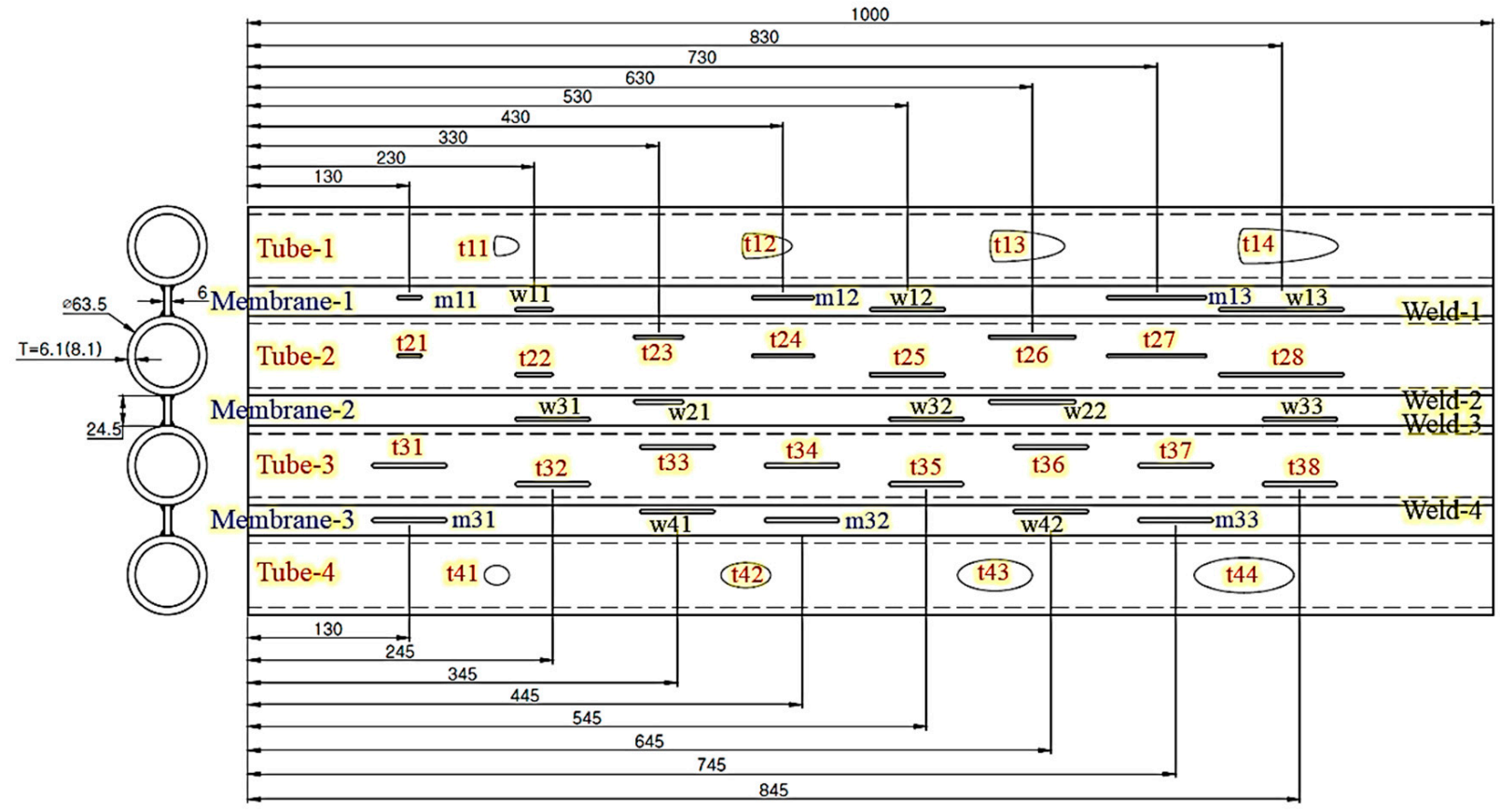

Figure 8. Specimen with different shape and size of artificial damages. 
Table 1. Specification of artificial taper-type wear on the Tube-1 and Tube-4.

\begin{tabular}{cccccccccc}
\hline$\#$ & $\begin{array}{c}\text { Width } \\
{[\mathrm{mm}]}\end{array}$ & $\begin{array}{c}\text { Length } \\
{[\mathrm{mm}]}\end{array}$ & $\begin{array}{c}\text { Depth } \\
(\mathbf{m m})\end{array}$ & $\begin{array}{c}\text { Position } \\
(\mathbf{m m})\end{array}$ & $\#$ & $\begin{array}{c}\text { Width } \\
{[\mathrm{mm}]}\end{array}$ & $\begin{array}{c}\text { Length } \\
{[\mathrm{mm}]}\end{array}$ & $\begin{array}{c}\text { Depth } \\
(\mathbf{m m})\end{array}$ & $\begin{array}{c}\text { Position } \\
(\mathbf{m m})\end{array}$ \\
\hline \multicolumn{7}{c}{ Tube-1 (One-Side Taper-Type Wear) } & \multicolumn{3}{c}{ Tube-4 (Two-Side Taper-Type Wear) } \\
\hline $\mathrm{t} 11$ & 16 & 20 & 0.9 & 200 & $\mathrm{t} 41$ & 16 & 20 & 0.9 & 200 \\
\hline $\mathrm{t} 12$ & 20 & 40 & 1.5 & 400 & $\mathrm{t} 42$ & 20 & 40 & 1.5 & 400 \\
\hline $\mathrm{t} 13$ & 25 & 60 & 2.5 & 600 & $\mathrm{t} 43$ & 25 & 60 & 2.5 & 600 \\
\hline $\mathrm{t} 14$ & 28 & 80 & 3.1 & 800 & $\mathrm{t} 44$ & 28 & 80 & 3.1 & 800 \\
\hline
\end{tabular}

Table 2. Specification of artificial slit-type damages on the Tube-2 and Tube-3.

\begin{tabular}{cccccccccc}
\hline$\#$ & $\begin{array}{c}\text { Width } \\
{[\mathbf{m m}]}\end{array}$ & $\begin{array}{c}\text { Length } \\
{[\mathbf{m m}]}\end{array}$ & $\begin{array}{c}\text { Depth } \\
(\mathbf{m m})\end{array}$ & $\begin{array}{c}\text { Position } \\
(\mathbf{m m})\end{array}$ & $\#$ & $\begin{array}{c}\text { Width } \\
{[\mathbf{m m}]}\end{array}$ & $\begin{array}{c}\text { Length } \\
{[\mathbf{m m}]}\end{array}$ & $\begin{array}{c}\text { Depth } \\
(\mathbf{m m})\end{array}$ & $\begin{array}{c}\text { Position } \\
(\mathbf{m m})\end{array}$ \\
\hline \multicolumn{7}{c}{ Tube-2 (Same Width) } \\
$\mathrm{t} 21$ & 7 & 20 & 0.9 & 130 & $\mathrm{t} 31$ & 7 & 60 & 0.23 & 130 \\
\hline $\mathrm{t} 22$ & 7 & 30 & 0.9 & 230 & $\mathrm{t} 32$ & 7 & 60 & 0.50 & 245 \\
\hline $\mathrm{t} 23$ & 7 & 40 & 0.9 & 330 & $\mathrm{t} 33$ & 7 & 60 & 0.96 & 345 \\
\hline $\mathrm{t} 24$ & 7 & 50 & 0.9 & 430 & $\mathrm{t} 34$ & 7 & 60 & 1.08 & 445 \\
\hline $\mathrm{t} 25$ & 7 & 60 & 0.9 & 530 & $\mathrm{t} 35$ & 7 & 60 & 1.5 & 545 \\
\hline $\mathrm{t} 26$ & 7 & 70 & 0.9 & 630 & $\mathrm{t} 36$ & 7 & 60 & 1.86 & 645 \\
\hline $\mathrm{t} 27$ & 7 & 80 & 0.9 & 730 & $\mathrm{t} 37$ & 7 & 60 & 2.34 & 745 \\
\hline $\mathrm{t} 28$ & 7 & 100 & 0.9 & 830 & $\mathrm{t} 38$ & 7 & 60 & 2.64 & 845 \\
\hline
\end{tabular}

The tubes were welded with a $6.0 \mathrm{~mm}$ thick membrane. There six slit-type artificial damages (w11, w12, w13, w31, w31, w33) on the two Membrane -1 and -2 . The damages have the same width of $7.0 \mathrm{~mm}$, different lengths from 20 to $80 \mathrm{~mm}$, and different depths from 0.3 to $2.4 \mathrm{~mm}$, as shown in Table 3. On the four welding lines (Welds 1, 2, 3, and 4), there are ten slit-type artificial damages (w11-w42) with the same width of $7.0 \mathrm{~mm}$, different lengths from 30 to $100 \mathrm{~mm}$, and different depths from 0.6 to $3.0 \mathrm{~mm}$, as shown in Table 4 . Totally, there are 40 artificial damages produced on the tubes, membranes, and welding lines. The picture of the specimen with damages is shown in Figure 9.

Table 3. Specification of artificial slit-type damages on the Membrane-1 and Membrane-3.

\begin{tabular}{cccccccccc}
\hline$\#$ & $\begin{array}{c}\text { Width } \\
{[\mathbf{m m}]}\end{array}$ & $\begin{array}{c}\text { Length } \\
{[\mathbf{m m}]}\end{array}$ & $\begin{array}{c}\text { Depth } \\
(\mathbf{m m})\end{array}$ & $\begin{array}{c}\text { Position } \\
\mathbf{( m m})\end{array}$ & $\#$ & $\begin{array}{c}\text { Width } \\
{[\mathbf{m m}]}\end{array}$ & $\begin{array}{c}\text { Length } \\
{[\mathbf{m m}]}\end{array}$ & $\begin{array}{c}\text { Depth } \\
(\mathbf{m m})\end{array}$ & $\begin{array}{c}\text { Position } \\
(\mathbf{m m})\end{array}$ \\
\hline $\mathrm{m} 11$ & 7 & 20 & 0.9 & 130 & $\mathrm{~m} 31$ & 7 & 60 & 0.3 & 130 \\
\hline $\mathrm{m} 12$ & 7 & 50 & 0.9 & 430 & $\mathrm{~m} 32$ & 7 & 60 & 1.2 & 445 \\
\hline $\mathrm{m} 13$ & 7 & 80 & 0.9 & 730 & $\mathrm{~m} 33$ & 7 & 60 & 2.4 & 745 \\
\hline
\end{tabular}


Table 4. Specification of slit-type damages on the Weld-1 4.

\begin{tabular}{cccccccccc}
\hline$\#$ & $\begin{array}{c}\text { Width } \\
{[\mathbf{m m}]}\end{array}$ & $\begin{array}{c}\text { Length } \\
{[\mathbf{m m}]}\end{array}$ & $\begin{array}{c}\text { Depth } \\
(\mathbf{m m})\end{array}$ & $\begin{array}{c}\text { Position } \\
(\mathbf{m m})\end{array}$ & $\#$ & $\begin{array}{c}\text { Width } \\
{[\mathbf{m m}]}\end{array}$ & $\begin{array}{c}\text { Length } \\
{[\mathbf{m m}]}\end{array}$ & $\begin{array}{c}\text { Depth } \\
(\mathbf{m m})\end{array}$ & $\begin{array}{c}\text { Position } \\
(\mathbf{m m})\end{array}$ \\
\hline $\mathrm{w} 11$ & 7 & 30 & 0.9 & 230 & w31 & 7 & 60 & 0.6 & 245 \\
\hline $\mathrm{w} 12$ & 7 & 60 & 0.9 & 530 & w32 & 7 & 60 & 1.5 & 545 \\
\hline $\mathrm{w} 13$ & 7 & 100 & 0.9 & 830 & w33 & 7 & 60 & 3.0 & 845 \\
\hline & & Weld-2 & & & & & Weld-4 & & \\
\hline w21 & 7 & 40 & 0.9 & 330 & w41 & 7 & 60 & 0.9 & 345 \\
\hline w22 & 7 & 70 & 0.9 & 630 & w42 & 7 & 60 & 1.8 & 645 \\
\hline
\end{tabular}

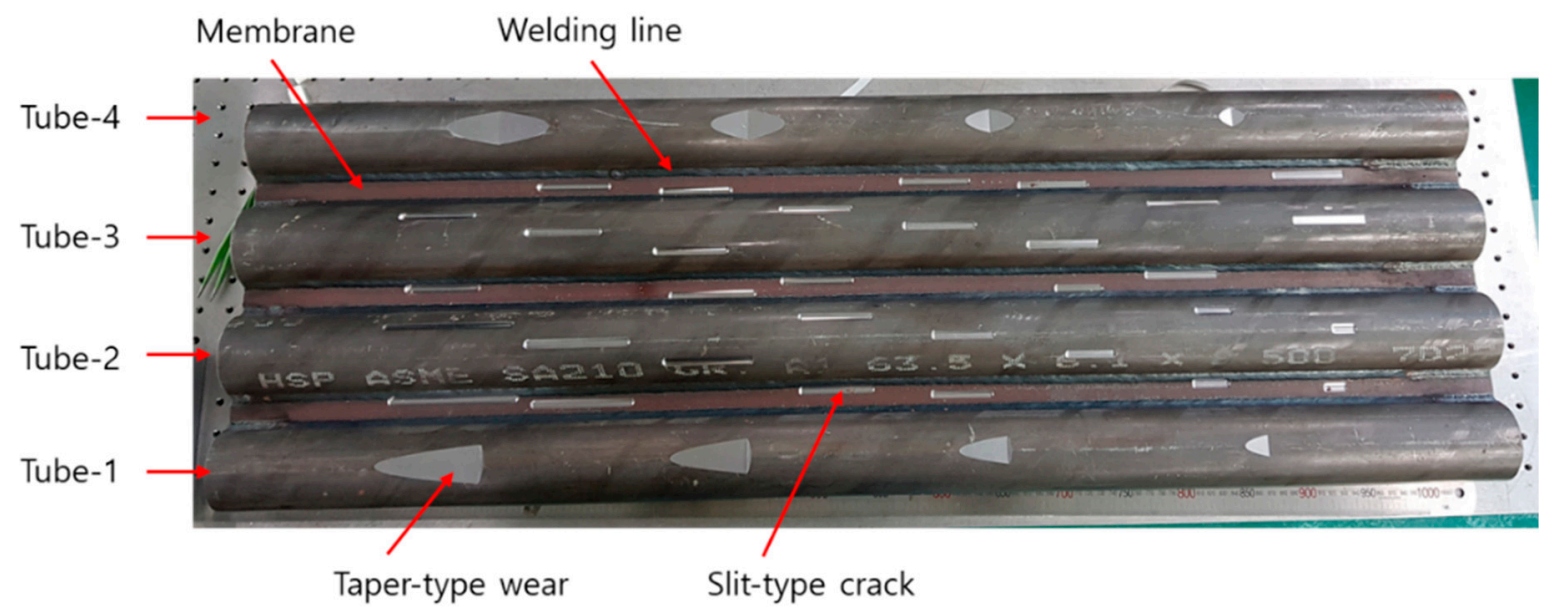

Figure 9. Sample specimens with four water-cooled tubes and artificial damages.

\subsection{Inspection System}

Figure 10 shows the prototype of the inspection system. In the magnetic leakage testing (MFLT) module, the magnetizer has a pole distance of $15 \mathrm{~mm}$ and has manufactured the profile following the tube and membrane surfaces. It maintains about $1.0 \mathrm{~mm}$ of distance above the specimen surface by the support of the three wheels. The magnetizer has 100 turns of copper wire and supplied by a current of about $200 \mathrm{~mA}$ to produce a magnetic field into the specimen. There are 48 Hall sensors arrayed at an interval of $2.5 \mathrm{~mm}$ on a curve following the tube and membrane surfaces. The MFLT probe scanned the specimen with steps of $4.0 \mathrm{~mm}$. In the FUP, there are 6 flexible ultrasound transducers having a center frequency of $5 \mathrm{MHz}$. The MFLT module, including the magnetizer and magnetic sensor array, can be exchanged with the FUP module. The measured signal can be processed and displayed in real-time in a LabVIEW software on a notebook. 


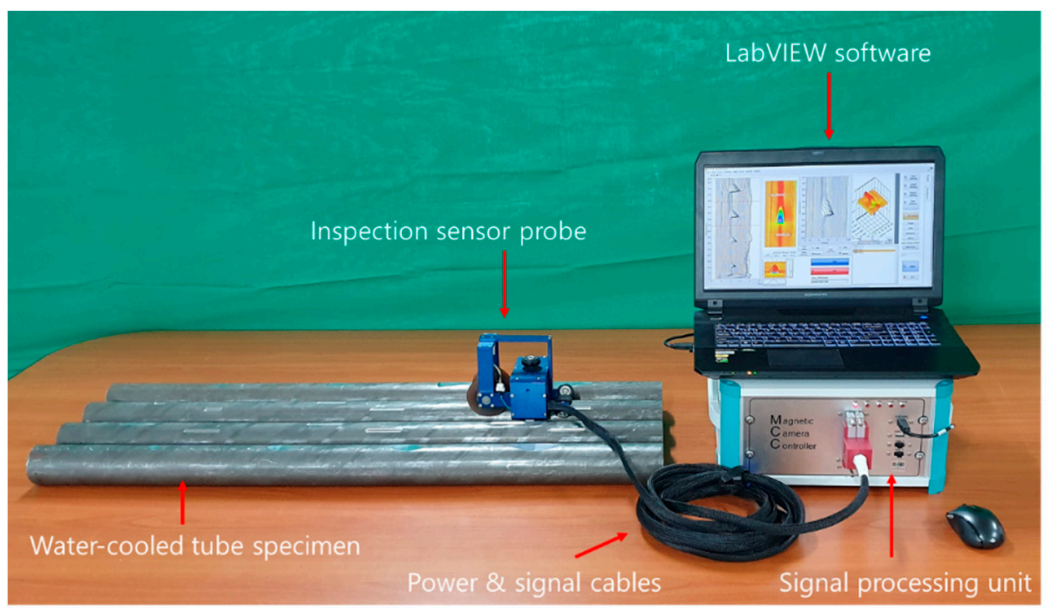

Figure 10. The prototype of the inspection system for water-cooled tubes.

\subsection{Experiment Results}

Figure 11 shows the scan results of the MFLT module on the Tube-1. Magnetic field distribution around the damages could help to recognize the presence of the damages. All the taper-type wears ( $\mathrm{t} 11, \mathrm{t} 12, \mathrm{t} 13$, and $\mathrm{t} 14)$ could be detected, and the magnetic field intensity increases as the size of the wear increases. In addition, the slit-type damages on the membrane ( $\mathrm{m} 11, \mathrm{~m} 12$, and $\mathrm{m} 13$ ) could also be detected, but the damages on the welding line (w11, w12, and w13) were out of the sensing area. The smallest slit-type damage (m11) has a length of $20 \mathrm{~mm}$, depth of $0.9 \mathrm{~mm}$, and $7 \mathrm{~mm}$ width that could be detected. Similarly, the taper-type wears on Tube- $4(\mathrm{t} 41, \mathrm{t} 42, \mathrm{t} 43$, and $\mathrm{t} 44)$ could be detected, as shown in Figure 12. However, the smallest size of slit-type damage on Membrane-3 (m31) having a length of $60 \mathrm{~mm}$, depth of $0.3 \mathrm{~mm}$, and width of $7 \mathrm{~mm}$ could not be detected; this is because the damage has a smallest depth of $0.3 \mathrm{~mm}$. The damages (m32 and m33) which have depths of $1.2 \mathrm{~mm}$ and $2.4 \mathrm{~mm}$, could be detected. The slit-type damages on the Weld-4 (w41 and w42) were out of the sensing area, but a part of the w42 signal could be measured because the damage has the deepest depth of $1.8 \mathrm{~mm}$.
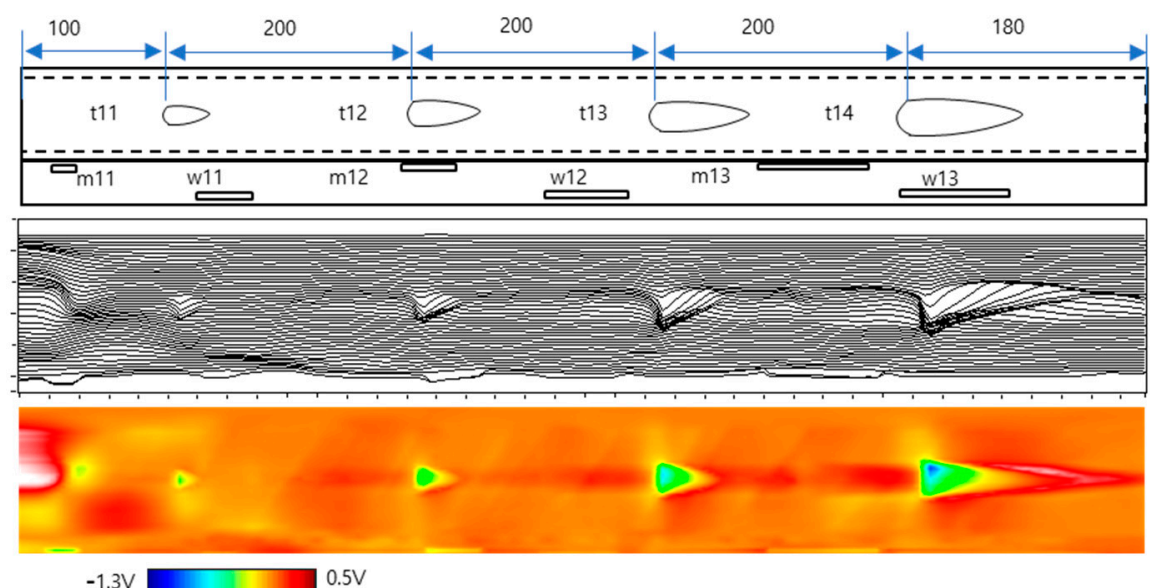

Figure 11. Distribution of magnetic field on the Tube-1, Membrane-1, and Weld-1 having artificial taper-type wear (single side) and slit-type damages. 

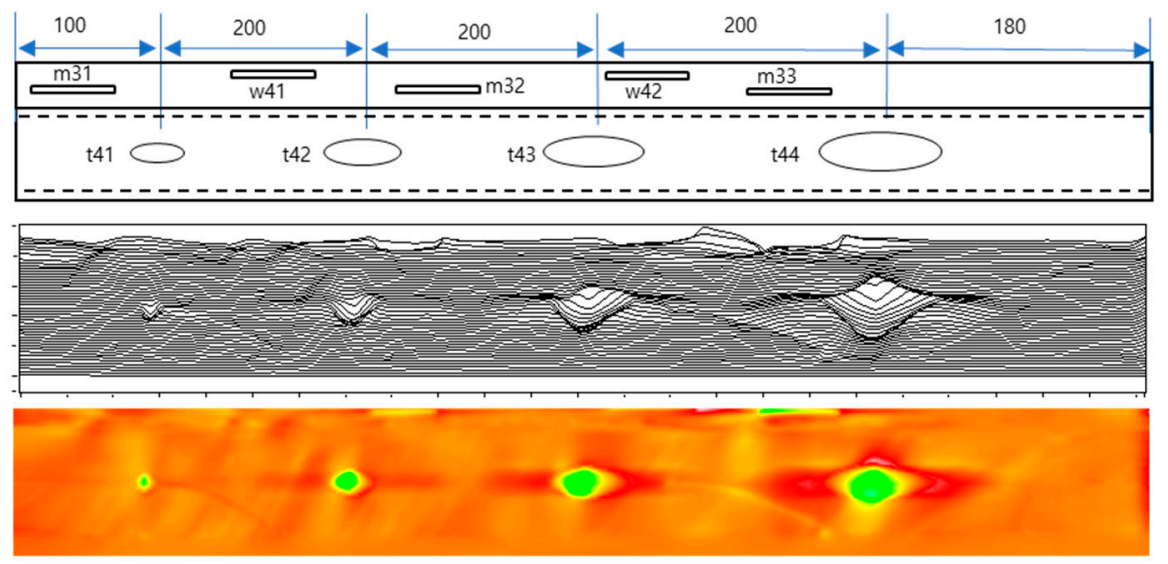

$-1.3 \mathrm{~V}$

$0.5 \mathrm{~V}$

Figure 12. Distribution of magnetic field on the Tube-4, Membrane-3, and Weld-4 having artificial taper-type wear (double side) and slit-type damages.

Figures 13 and 14 show the detection result of the slit-type damages. All defects ( $\mathrm{t} 21 \sim \mathrm{t} 28, \mathrm{t} 31 \sim \mathrm{t} 38)$ with a depth of $0.3 \mathrm{~mm}$ or more located in Tube- 2 and Tube- 3 could be detected. In addition, damages of $0.9 \mathrm{~mm}$ in depth and $30 \mathrm{~mm}$ in length (w11) or more were detected in Weld-1 could also be detected. However, damages (w21, w22, w31, w32, w33) located in Weld-2 and Weld-3 were difficult to detect. Nevertheless, damages with a depth of $0.9 \mathrm{~mm}$ or more in Weld-4 (w41, w42) and damages with a depth of $1.2 \mathrm{~mm}$ or more in Membrane-3 (m32, m33) could be detected. This is because that when the sensor for magnetic flux density measurement scans Tube-4, it is skewed toward Membrane-3, and the lift-offs of Membrane- 2 and Membrane- 3 are not the same.

From the above results, the depths of damages detectable in the tube and membrane are $0.3 \mathrm{~mm}$ and $0.9 \mathrm{~mm}$, respectively. In addition, some damages having a depth of $0.9 \mathrm{~mm}$ or more could be detected in the welded part due to the influence of the welding beads.

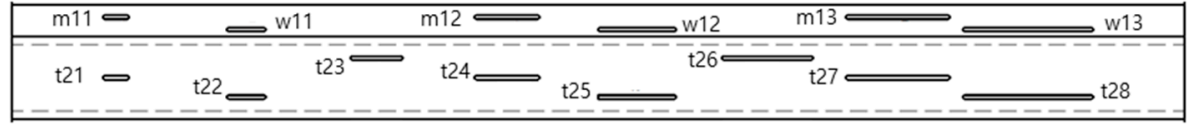
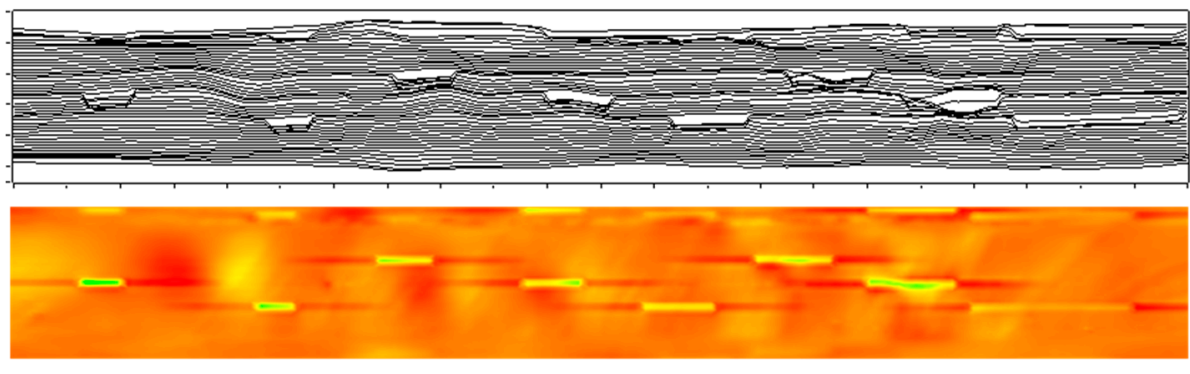

$-1.3 \mathrm{r}-0.5 \mathrm{~V}$

Figure 13. Distribution of magnetic field on the Tube-2, Membrane-1 (Weld-1, 2) having slit-type damages. 

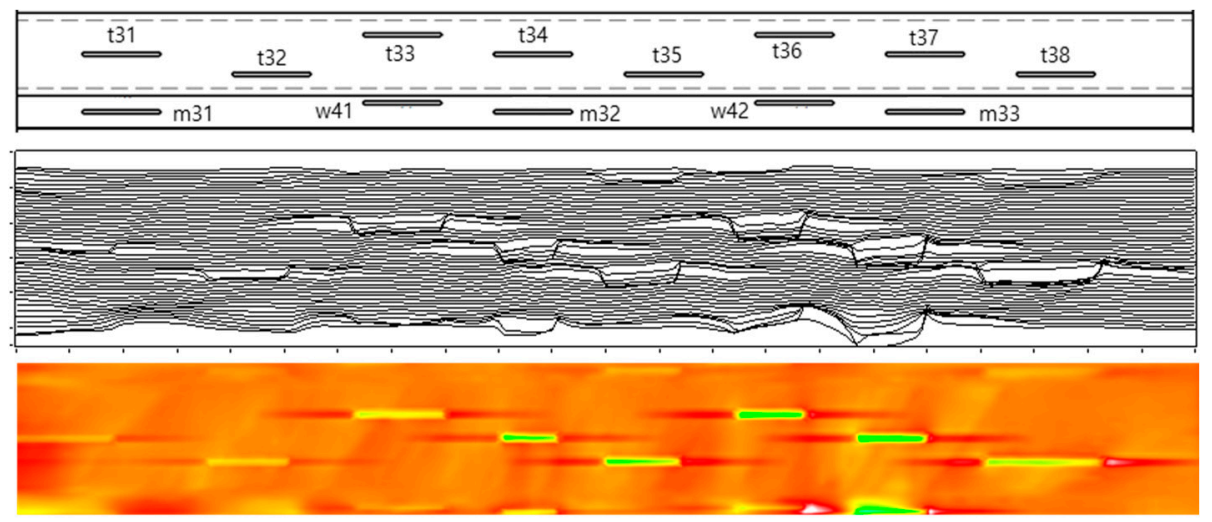

$-1.3 \mathrm{~V} \square 0.5 \mathrm{~V}$

Figure 14. Distribution of magnetic field on the Tube-3, Membrane-3, and Weld- 4 having slit-type damages.

Figure 15 shows the graph showing the relationship measured data with the depth of the defect on Tube-3. The damages have the same width of $7 \mathrm{~mm}$ and length of $60 \mathrm{~mm}$. The measured data is the minimum data points selected from the numbers of Hall sensor array that are on the damages during the scan. There are 30 sensors, and 15 sensors data plotted on Figure 15a,b. The data in Figure 15a has more noise than in Figure 15b because some sensors are located far from the damages. Then, data of 15 sensors is used for further evaluation of the damages' depth. The average data of the sensors are used to reduce noise that may occur rather than a single sensor. Also, the relationship between the measured data with the damages' depth is expressed in Equation (13). This form of the experimental equation is same as the theoretical analysis by the dipole model of the previous section (Equation (9)). The factors $c_{1}, c_{2}$, and $a_{2}$ are $121.08,6.65$, and 2.52, respectively.

$$
d=\sqrt{121.08 V_{H}+6.65}+2.52
$$

a)

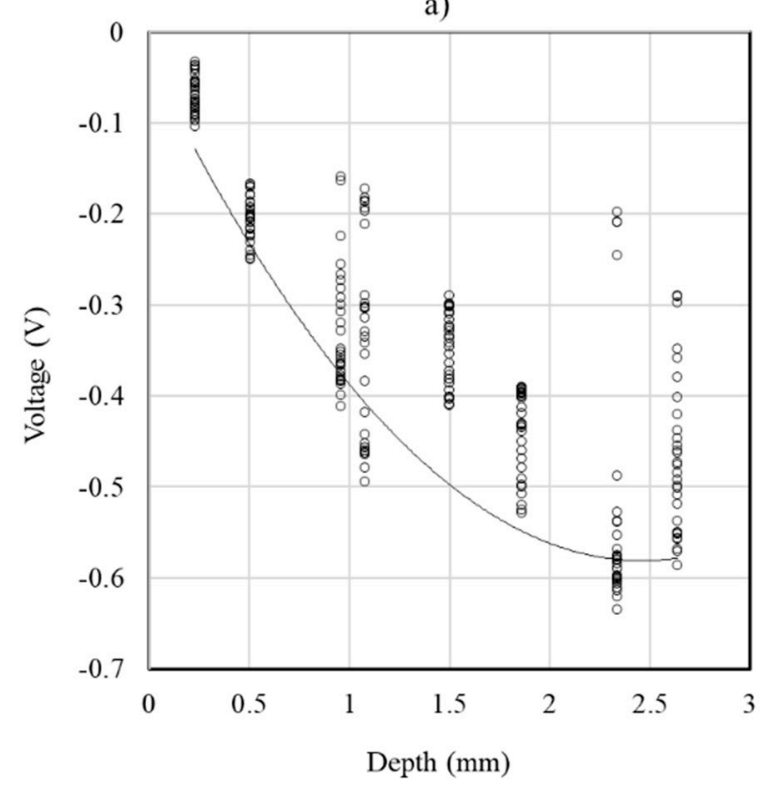

b)

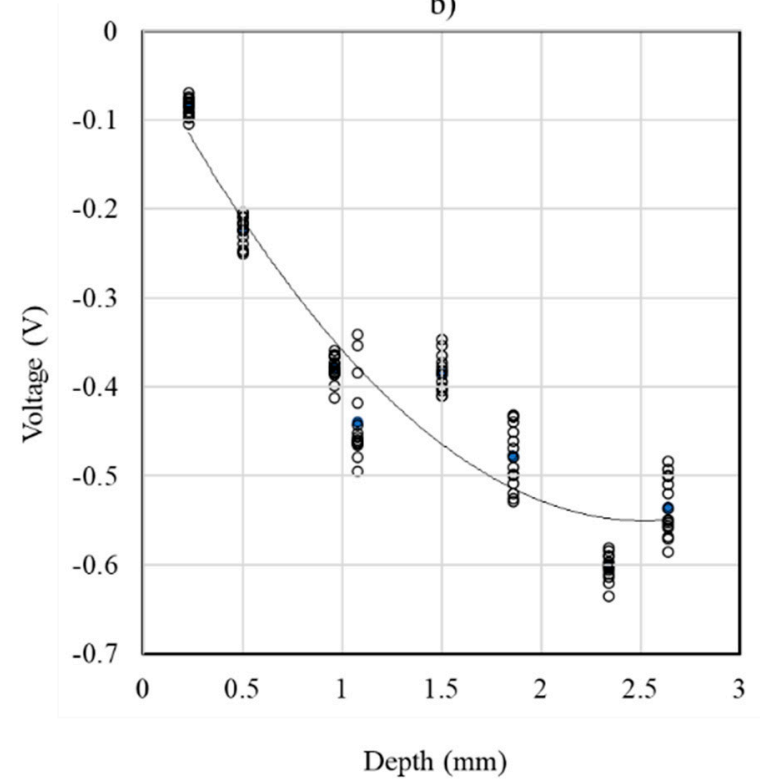

Figure 15. Relationship between the depth of flaw and the measured data with magnetic flux density method: (a) data of 30 sensors and (b) data of 15 sensors. 
From Equation (13), the depth of damages on the tube $(\bigcirc)$, membrane ( $\square$ ), and weld $(\Delta)$ were estimated, as shown in Figure 16. The standard deviations of the depth estimation are $0.329,0.269$, and $0.523 \mathrm{~mm}$ for the damages on the tube, membrane and weld, respectively. The best estimation result is for damages on the membrane because the surface specimen is flat. The worst case for the damages on weld were due to the roughness of the weld surface, the sensor lift-off variation due to welding bead, and the edge effect at the terminal of the magnetizer.

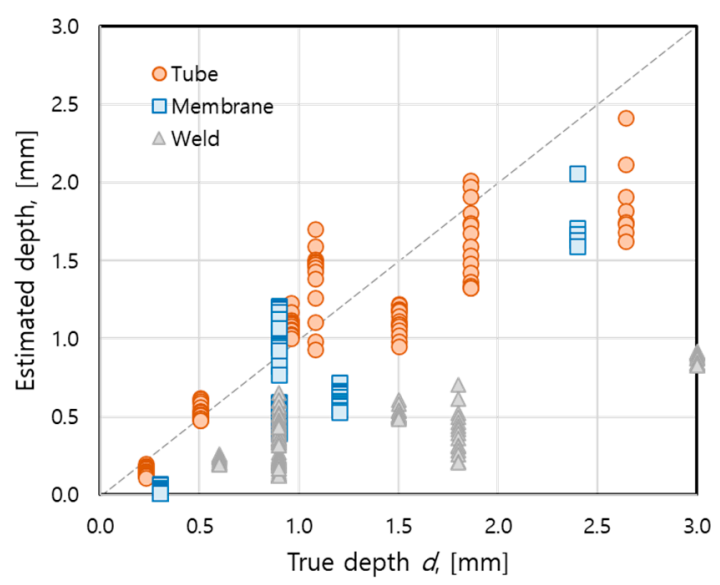

Figure 16. Estimation of depth of damages on the tube, membrane, and weld.

Figure 17 shows the B-scan result measured by the FUP after filling the acoustic medium and wrapping it with PET to the damage of Tube- 1 (t11 t14). The horizontal axis represents time $\left(T_{i}\right)$, and the vertical axis represents the moving distance of the FUP. The position of $T_{1}$ for each movement distance was about $20 \mu$ s before the start of the scan, but after $550 \mathrm{~mm}$, it appeared at 17-18 $\mu \mathrm{s}$. This is because of the variation of the inclined angle of the FUP and deformation of the membrane due to variation of the FUP lift-off. Therefore, it is necessary to shift the flying time on specimen surface $\left(T_{1}\right)$ for each scan position, as expressed in Equation (14). Furthermore, it can be seen that in the vicinity of $100,300,525$, and $725 \mathrm{~mm}$, the delay of the FUP signal is longer than that of other locations, and near-side damage occurs in the corresponding region. It can also be determined from the delay of the signal that the shape of the damage is inclined to one side, and the depth can be estimated.

$$
\left[\vec{T}_{1}\right]=[\overrightarrow{0}]
$$

Figure 18 shows the B-scan results of the FUP measured from the back surface of the damages (m31-33) of Membrane-3 and (w21, w22) of Weld-2 using FUP. Similar to the previous experimental results. The position of $\mathrm{T}_{1}$ for each movement distance was about $22 \mu \mathrm{s}$ before the start of the scan, but after $450 \mathrm{~mm}$ past $\mathrm{m} 32$, it is back to $21 \mu \mathrm{s}$. Unlike the case of the near-side damage in Figure 17, it is possible to recognize that there is no near-side damage because the FUP signal appears continuously. On the other hand, it is observed that $\mathrm{m} 31, \mathrm{~m} 32$, and $\mathrm{m} 33$ damages occur around $130 \mathrm{~mm}, 460 \mathrm{~mm}$, and $750 \mathrm{~mm}$, respectively. In addition, signal attenuation appears in the range of $625-700 \mathrm{~mm}$. This is because the ultrasonic wave attenuates at the edge of the weld defect w 42 located in Membrane-3. A similar phenomenon occurred near the weld defect (w41) at 325-380 mm. The depth of the damages was estimated, as shown in Figure 19. The standard deviation of the depth estimation is about $0.089 \mathrm{~mm}$, which is much more accurate than using the magnetic flux leakage testing method. 


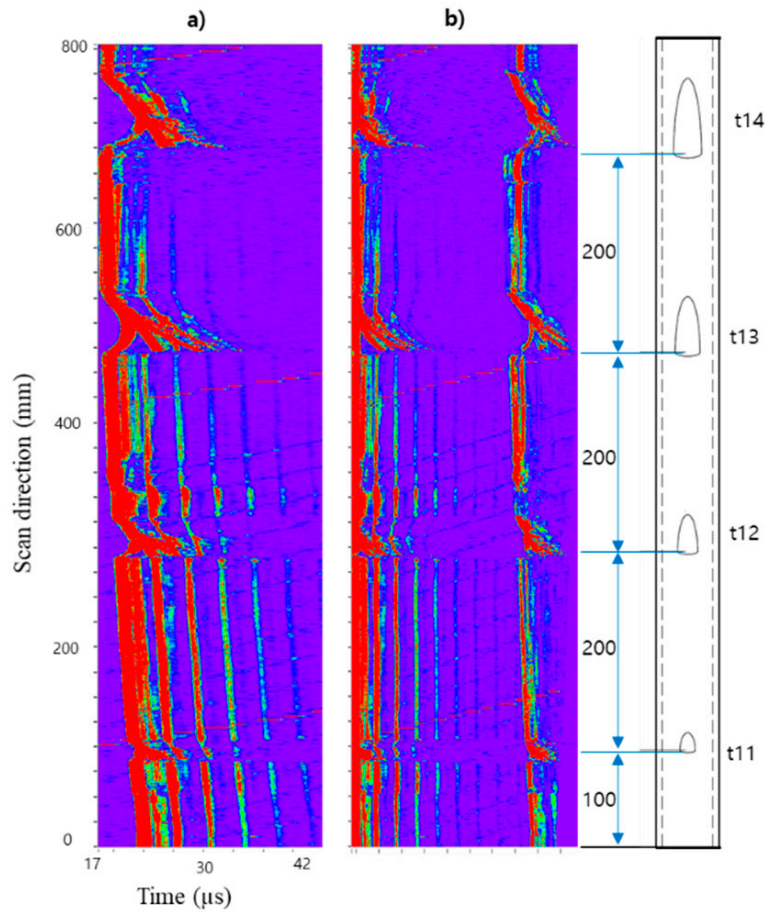

Figure 17. B-Scan results with FUP on the near-side damages of Tube-1 (a) before and (b) after $T_{1}$ adjustment.

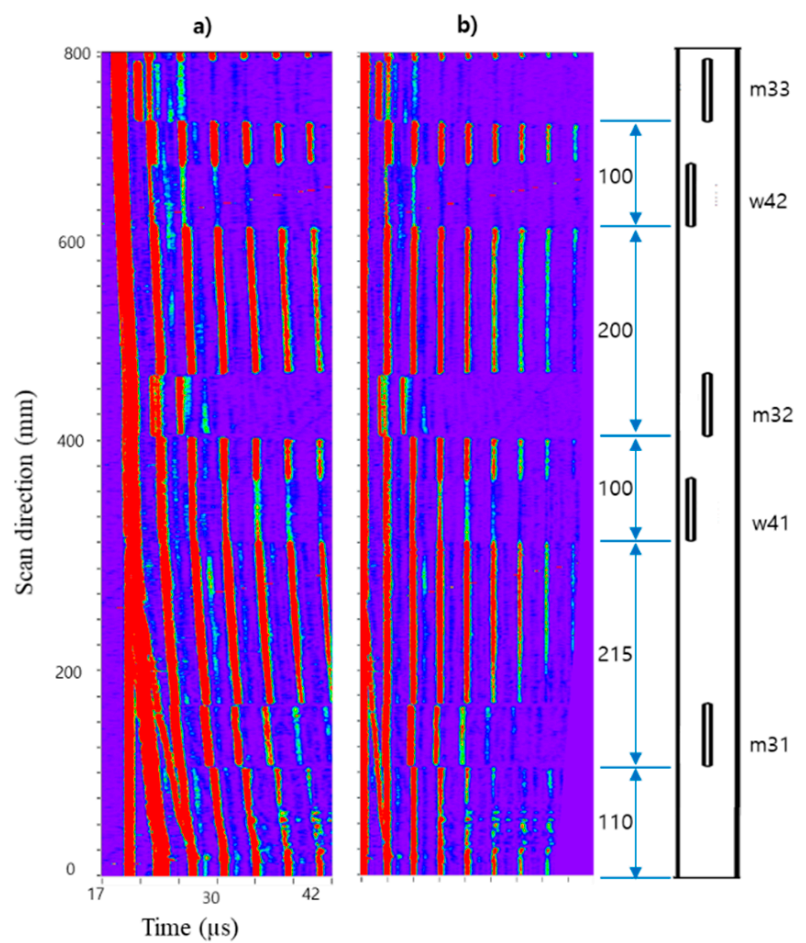

Figure 18. B-Scan results with FUP on the far-side damages of Membrane-3 and Weld-2 (a) before and (b) after $T_{1}$ adjustment. 


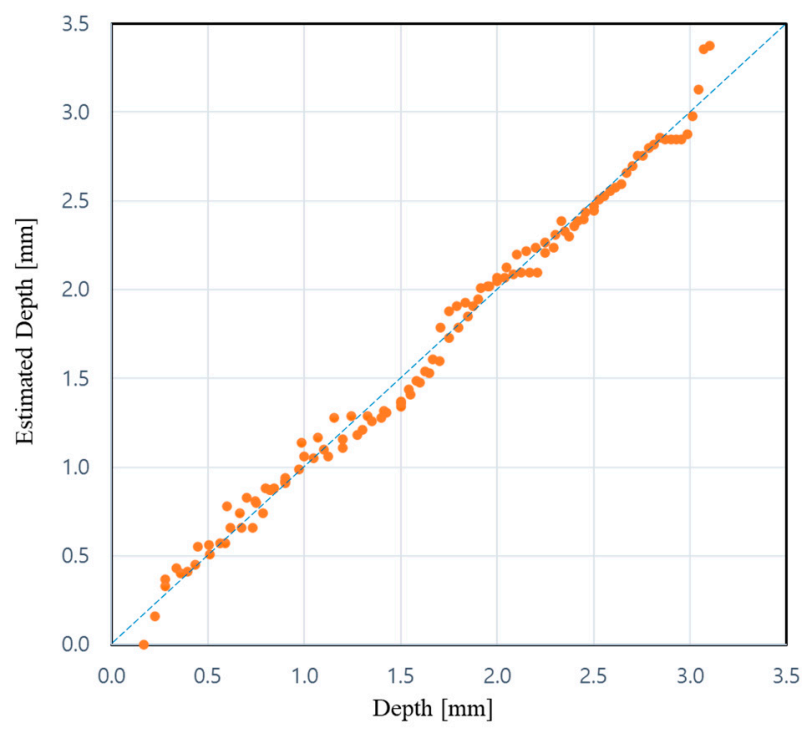

Figure 19. Estimation of damages' depth using the FUP.

\section{Conclusions}

In this paper, a nondestructive inspection system was proposed to detect defects on the near-side and far-side of the boiler water-cooled wall tube, membrane, and weld and to quantitatively evaluate the size of the defects. A magnetizer manufactured in a curved shape according to the cross-sectional shape of the tube, membrane, and welding part magnetizes a portion of the water-cooled wall in the axial direction. In addition, the shape of the surface defect can be qualitatively determined from the magnetic flux density distribution measured by the magnetic sensor array deflected from the center of the magnetizer to one side. The minimum depths of surface defects that can be measured are $0.3 \mathrm{~mm}, 0.9 \mathrm{~mm}$, and $1.2 \mathrm{~mm}$ in each case of the tube, membrane, and weld. The depth of defects located in the tube, membrane, and weld can be quantitatively evaluated with a standard deviation of $0.329,0.269$, and $0.523 \mathrm{~mm}$. A method of scanning with a flexible ultrasonic probe (FUP) after applying an acoustic medium to the defect surface of the water-cooled wall, covering a thin film of PET (polyethylene terephthalate), and applying a separate acoustic medium was proposed. According to the FUP arranged in a direction perpendicular to each cross-section of the tube, membrane, and weld, the location and shape of the surface defect and the back defect can be distinguished. Furthermore, the depth of the defect can be quantitatively evaluated with a standard deviation of $0.089 \mathrm{~mm}$.

By combination of the magnetic flux leakage testing and ultrasonic testing, both the near-side and far-side defects could be detected and a quantitative evaluation of the depth could be made. Furthermore, the system is also expected to detect and evaluate the internal surface defects. For instance, if the defect is shallow in the near-surface, then the magnetic flux leakage testing is efficient for detection; otherwise, if the defect is deep to near the far-side, then the ultrasonic is more efficient. The further development of the proposed system should quantitatively evaluate different sizes of the defect such as length and width, or recognize the shape of the defects.

Author Contributions: Conceptualization, M.L. and J.L.; methodology, M.L.; software, M.L. and C.-T.P.; validation, J.L., M.L. and E.C.; formal analysis, E.C.; investigation, J.L. and M.L.; resources, J.L. and M.L.; data curation, E.C. and M.L.; writing-original draft preparation, J.L.; writing-review and editing, M.L.; visualization, C.-T.P. and E.C.; supervision, J.L. and M.L.; project administration, M.L. and J.L.; funding acquisition, M.L. and J.L. All authors have read and agreed to the published version of the manuscript.

Funding: This work was supported by the Vietnam National Foundation for Science and Technology Development (NAFOSTED) under grant number 103.02-2019.342, and the National Research Foundation of Korea (NRF) grant funded by the Korea government (MSIT) (No.NRF-2019R1A2C2006064). 
Conflicts of Interest: The authors declare no conflict of interest.

\section{References}

1. Basu, P. Combustion of coal in circulating fluidized-bed boilers: A review. Chem. Eng. Sci. 1999, 54, 701-764. [CrossRef]

2. Wang, B. Erosion-corrosion of thermal sprayed coatings in FBC boilers. Wear 1996, 199, 24-32. [CrossRef]

3. Kim, T.-W.; Choi, J.-H.; Shun, D.-W.; Son, J.-E.; Jung, B.; Kim, S.-S.; Kim, S.-D. A Tube Thickness Map of Water Wall in a Commercial Circulating Fluidized Bed Combustor. Korean Chem. Eng. Res. 2005, 43, 412-418.

4. Jung, M.-J.; Park, B.-C.; Bae, J.-H.; Shin, S.-C. PAUT-based defect detection method for submarine pressure hulls. Int. J. Nav. Archit. Ocean Eng. 2018, 10, 153-169. [CrossRef]

5. Kim, Y.; Cho, S.; Park, I.K. Analysis of Flaw Detection Sensitivity of Phased Array Ultrasonics in Austenitic Steel Welds According to Inspection Conditions. Sensors 2021, 21, 242. [CrossRef] [PubMed]

6. Yusa, N.; Chen, Z.; Miya, K. Quantitative profile evaluation of natural defects in a steam generator tube from eddy current signals. Int. J. Appl. Electromagn. Mech. 2000, 12, 139-150. [CrossRef]

7. Xie, S.; Chen, Z.; Takagi, T.; Uchimoto, T. Evaluation of Wall Thinning in Carbon Steel Piping Based on Magnetic Saturation Pulsed Eddy Current Testing Method. Stud. Appl. Electromagn. Mech. 2013, 39, 296-303.

8. Le, M.; Kim, J.; Kim, J.; Do, H.S.; Lee, J. “Electromagnetic testing of moisture separator reheater tubes using a bobbin-type integrated Hall sensor array. Int. J. Appl. Electromagn. Mech. 2017, 55, S203-S209. [CrossRef]

9. Le, M.; Kim, J.; Kim, J.; Do, H.S.; Lee, J. Nondestructive testing of moisture separator reheater tubing system using Hall sensor array. Nondestruct. Test. Eval. 2018, 33, 35-44. [CrossRef]

10. Lafontaine, G.; Hardy, F.; Renaud, J. X-Probe®ECT array: A high-Speed Replacement for Rotating Probes. In Proceedings of the Third International Conference on NDE in Relation to Structural Integrity for Nuclear and Pressurized Components, Seville, Spain, 14-16 November 2001.

11. Joubert, P.Y.; Bihan, Y.L.; Placko, D. Localization of defects in steam generator tubes using a multi-coil eddy current probe dedicated to high speed inspection. NDT E Int. 2002, 35, 53-59. [CrossRef]

12. Choi, C.D.; Lim, I.S. Comparative Reliability of Nondestructive Testing for Weld: Water Wall Tube in Thermal Power Plant Boiler Case Study. J. Appl. Reliab. 2018, 18, 240-249. [CrossRef]

13. Vajpayee, A.; Russell, D. Automated Condition Assessment of Boiler Water Wall Tubes Using Remote Field Technology. A Revolution over Traditional and Existing Techniques. In Proceedings of the 10th International Conference of the Slovenian Society for Nondestructive Testing, Ljubljana, Slovenia, 1-3 September 2009; pp. 523-530.

14. Gil, D.-S.; Jung, G.-J.; Seo, J.-S.; Kim, H.-J.; Kwon, C.-W. Simulation of Remote Field Scanner for Defect Evaluation of Water Wall Tube within the Fluidized Bed Boiler. KEPCO J. Electr. Power Energy 2020, 6, 145-150.

15. Atherton, D.L.; Mackintosh, D.D.; Sullivan, S.P.; Dubois, J.M.S.; Schmidt, T.R. Remote-Field Eddy Current Signal Representation. Mater. Eval. 1993, 51, 782-789.

16. Park, J.-H.; Yoo, H.-R.; Kim, D.-K.; Kim, H.-J.; Cho, S.-H.; Song, S.-J.; Kim, H.-M.; Park, G.-S.; Rho, Y.-W. Development of RFECT System for In-Line Inspection Robot Considering Extendibility of Receiving Senosors based on Parallel Lock-in Amplifer. Int. J. Precis. Eng. Manuf. 2017, 18, 145-153. [CrossRef]

17. Park, J.W.; Park, J.H.; Song, S.J.; Kishore, M.B.; Kwon, S.G.; Kim, H.J. Enhanced Detection of Defects Using GMR Sensor Based Remote Field Eddy Current Techique. J. Magn. 2017, 22, 531-538. [CrossRef]

18. Lee, H.; Choe, E.; Lee, J.; Jung, G. Magnetic Flux Leakage Measurement System for Nondestructive Testing of Water-Cooled Wall. In Proceedings of the 2019 IEEE International Instrumentation and Measurement Technology Conference (I2MTC), Auckland, New Zealand, 20-23 May 2019; pp. 1-5.

19. Vakhguelt, A.; Kapayeva, S.D.; Bergander, M.J. Combination Non-Destructive Test (NDT) Method for Early Damage Detection and Condition Assessment of Boiler Tubes. Procedia Eng. 2017, 188, 125-132. [CrossRef]

20. Kapayeva, S.D.; Bergander, M.J.; Vakhguelt, A.; Khairaliyev, S.I. Remaining life assessment for boiler tubes affected by combined effect of wall thinning and overheating. Int. J. Vibroeng. 2017, 19, 5892-5907. [CrossRef]

21. Hwang, J.; Lee, J. Modeling of a Scan Type Magnetic Camera Image Using the Improved Dipole Model. J. Mech. Sci. Technol. 2006, 20, 1691-1701. [CrossRef]

22. Lee, J.; Jun, J.W.; Hwang, J.S.; Lee, S.H. Development of Numerical Analysis Software for the NDE by using Dipole Model. Key Eng. Mater. 2007, 353-358, 2383-2386. [CrossRef]

23. Le, M.; Kim, J.; Kim, S.; Lee, J. Nondestructive Testing of Pitting Corrosion Cracks in Rivet of Multilayer Structures. IJPEM 2016, 17, 1433-1442. [CrossRef] 